\title{
Added Value of Digital over Analog PET/CT: More Significant as Image Field of View and Body Mass Index Increase
}

\author{
Shirin Hatami, RT (R)(MR)(ARRT)(NMCT) ${ }^{1}$, Sarah Frye ${ }^{1}$, Anna McMunn², Crystal Botkin ${ }^{1}$, Razi Muzaffar ${ }^{3}$, \\ Kara Christopher ${ }^{4}$, and Medhat Osman ${ }^{3}$ \\ ${ }^{1}$ Doisy College of Health Sciences, Saint Louis University, St. Louis, Missouri; ${ }^{2}$ Saint Louis University Hospital, SSM Health, St. \\ Louis, Missouri; ${ }^{3}$ Division of Nuclear Medicine, Department of Radiology, Saint Louis University, St. Louis, Missouri; and ${ }^{4}$ Saint \\ Louis University Hospital, Cancer Center, St. Louis, Missouri
}

The digital PET/CT scanner with digital photon-counting technique promises a shorter scan time, improved small-lesion detectability, and reduced radiation dose for the PET and CT portions of the exam while improving image quality. Methods: In this single-institution retrospective review study, 84 participants who had undergone PET/CT on both analog and digital scanners were analyzed. The aim was to evaluate the impact of image field of view (FOV) and body mass index (BMI) on the digital compared with the analog PET/CT scanners. The participants were categorized into different groups based on their BMI. Total scan times, ${ }^{18} \mathrm{~F}-\mathrm{FDG}$ doses, and dose-length products (DLP) were collected and compared. Image quality was also assessed by certified nuclear medicine physicians and graded on a scale from 1 to 5. Results: In the skull-to-mid-thigh FOVs, the digital scanner had a scan time shorter by $37 \%(P<0.001)$, a ${ }^{18} \mathrm{~F}-\mathrm{FDG}$ dose lower by $16 \%(P<0.001)$, but only an $8 \%$ reduction in DLP $(P=$ 0.2). In the head-to-toe FOV cases, the digital scanner showed reductions in scan time $(33 \% ; P<0.001),{ }^{18} \mathrm{~F}-\mathrm{FDG}$ dose $(13 \% ; P$ $<0.001)$, and DLP (19\%; $P<0.001)$. When BMI was accounted for, the digital scanner had a scan time shorter by $33 \%(P<$ $0.001)$, as well as a reduced DLP $(P<0.001)$ and ${ }^{18} \mathrm{~F}-\mathrm{FDG}$ dose $(P<0.001)$, with the most prominent changes being in the overweight and obese participants. Image quality was also improved by the digital scanner, with a score of 4.5 , versus 4.0 for the analog scanner. Conclusion: The digital scanner has a shorter scan time and lower DLP, requires a lower ${ }^{18} \mathrm{~F}-\mathrm{FDG}$ dose, and provides improved image quality when compared with the analog scanner. The most impactful difference in scan time, DLP, and ${ }^{18} \mathrm{~F}-\mathrm{FDG}$ dose were observed in obese and overweight participants.

Key Words: scan time; DLP; weight-based dosing; BMI; digital

J Nucl Med Technol 2020; 48:354-360

DOI: 10.2967/jnmt.120.244160

\footnotetext{
Received Feb. 25, 2020; revision accepted Jul. 9, 2020

For correspondence or reprints contact: Shirin Hatami, Doisy College of Health Sciences, Saint Louis University, 1 N. Grand Blvd., St. Louis, MO 63103.

E-mail: shirin.hatami019@gmail.com

Published online Sep. 4, 2020.

COPYRIGHT (c) 2020 by the Society of Nuclear Medicine and Molecular Imaging.
}

$\mathbf{P}$ modalities and provides elaborate information at the cellular and molecular levels (1). Despite the impact of PET/CT on staging, restaging, and posttreatment evaluation in oncology, ionizing radiation has always been a concern. Efforts to reduce radiation exposure to both patients and staff have been ongoing in the imaging community (2). Advances in digital PET scanner detectors from conventional photomultiplier tubes to one-to-one solid detectors, and improvements in image reconstruction, are some changes taken toward reducing radiation dose in PET/CT imaging systems (3). The PET/CT scan creates high-resolution images while integrating the anatomic framework from CT with the functional images that come from PET.

Philips digital and analog PET/CT scanners were the focus of this study. Components of the analog PET scanner (Gemini) detectors include crystals that can convert photons to flashes of light but cannot count all individual photons. These scintillation crystals are coupled with multiple photomultiplier tubes that convert the flashes of light to electron signals. These electron signals are then sent to a computer for further processing and image production. The digital PET scanner (Vereos) is equipped with digital solid-state counting detectors that count every individual photon created during a PET scan (4,5). Each detector comprises multiple scintillator elements. The high count-rate ability of solid-state detectors comes from one-to-one coupling between the scintillator elements with light-sensing elements, leading to better spatial resolution and faster image acquisition (4).

To investigate the radiation dose in the CT part of the exam, dose-length product (DLP) is used-the total amount of radiation a person receives during a CT examination. On the digital scanner, DLP is the sum of the radiation dose from scout and slices; on the analog scanner, DLP is the radiation exposure from slices only. The digital scanner has promised a shorter scan time, a lower radiation dose from CT, and a lower ${ }^{18} \mathrm{~F}-\mathrm{FDG}$ dose (6). Since both the digital and the analog systems are used clinically, the possible impact of image field of view (FOV) and body mass index (BMI) in these 2 systems was considered. The purpose 
of this study was to compare the scan time and radiation dose associated with the digital and analog PET/CT systems. More effectively, the impact of image FOV and BMI on scan time and radiation dose was considered.

\section{MATERIALS AND METHODS}

The study was approved by the university's Institutional Review Board, and the requirement to obtain informed consent was waived.

\section{Participant Characteristics}

This study was a single-institution retrospective study of 84 PET/CT patients in an oncology cohort over a period of $5 \mathrm{y}$. The participants' age ranged between 18 and $75 \mathrm{y}$ old, and their BMI ranged between 15 and 68 .

In total, 110 participants had undergone both the analog and the digital PET/CT scans using the same injected ${ }^{18} \mathrm{~F}-\mathrm{FDG}$ dose of $5.18 \mathrm{MBq}(0.14 \mathrm{mCi} / \mathrm{kg})$, which was the factory-recommended dose. A maximum dose of $555 \mathrm{MBq}(15 \mathrm{mCi})$ for the analog scanner and a maximum dose of $370 \mathrm{MBq}(10 \mathrm{mCi})$ for the digital scanner were used; these were also factory-recommended by Philips. Given the retrospective nature of the study, the 2 scans could not be done on the same day. The time difference between the analog and the digital scans averaged $11 \mathrm{mo}$.

To calculate BMI in this study, participants' weight in kilograms was divided by the square of their height in meters $\left(\mathrm{kg} / \mathrm{m}^{2}\right)$. To factor changes in the ${ }^{18} \mathrm{~F}$-FDG dose based on significant weight changes, participants with greater than a $10 \%$ variation in their BMI during their follow-up period $(n=26)$ were excluded from further analyses. The remaining 84 cases (16 skull to mid thigh and 68 head to toe) were categorized into different groups based on BMI: underweight $(<18.9)$, normal weight (19$24.9)$, overweight (25-29.9), and obese ( $>30)$. All participants were instructed to fast for at least $6 \mathrm{~h}$ before the scan. The participants' blood glucose level was measured before injection of the ${ }^{18} \mathrm{~F}$-FDG dose, with an acceptable level being less than $200 \mathrm{mg} / \mathrm{dL}$. Participants were positioned in a quiet, dimly lit room and kept in a warm, unstimulated condition during their standard 60-min uptake time before imaging.

More recently, we adjusted the ${ }^{18} \mathrm{~F}-\mathrm{FDG}$ dosing in our center from weight-based to BMI-based and included 20 patients who had a prior scan using weight-based dosing. The patients were divided into 3 groups: BMIs of 25 or less, 26-34, and 35 or more.

Image quality was reviewed and analyzed by 2 board-certified nuclear medicine physicians who did not know the type of scanner used. Image quality for all scans was graded on a scale of 1 to $5(1=$ poor; 5 = excellent). Lesion detectability could not be assessed because of the differences in time between the scans.

\section{Image Data Acquisition}

Both PET/CT scanners used in this study are fused with a 64-slice CT scanner (7). The Gemini and the Vereos were used as the analog and digital scanners, respectively. Both were American College of Radiology-certified to ensure accurate analysis. For the Gemini (installed at this site in 2006), the CT image data were reconstructed using filtered backprojection technique, a $512 \times 512$ matrix, a $600-\mathrm{mm}$ FOV in participants with a BMI of less than 34 , and a 700-mm FOV in participants with a BMI of more than 34 .

To provide significant improvements in image quality combined with dose reduction capabilities, the Vereos (installed at this site in 2018) uses iDose technique to reconstruct CT images (7). iDose is the fourth generation of advanced iterative reconstruction technique and the latest addition to Philips DoseRight tools (7). The FOV for the different BMIs and the matrix size for the digital scanner were similar to those for the analog scanner. The CT scan was performed in a transaxial FOV with similar parameters for all participants regardless of BMI: $120 \mathrm{kVp}$, variable mAs in a range of 30-100, an average 15.5-cm axial FOV for the skull to the mid thigh and a 56.2- $\mathrm{cm}$ transaxial FOV for the whole body, a 4-mm slice thickness, a 4-mm increment, a pitch of 0.704 , a rotation time of $0.5 \mathrm{~s}$, and a collimation of $64 \times 0.625$. The iDose technique was applied for the digital scanner. The CT studies were obtained with an average time of $34.2 \mathrm{~s}$ for the skull to the mid thigh and $54.3 \mathrm{~s}$ for the whole body.

Ordered-subset expectation maximization was used in PET image reconstruction for both the digital and the analog scanners ( 3 iterations with 33 subsets for the analog scanner and 3 iterations with 15 subsets for the digital scanner). The PET parameters included 10 frames for the skull to the mid thigh and a maximum of 18 frames for the whole body. In the analog PET exams, photon counting time per frame varied with BMI: for a BMI of less than $25,60 \mathrm{~s}$ per frame for the skull to the mid thigh (frames 1-10) and $30 \mathrm{~s}$ per frame for the lower extremity (frames 11-18); for a BMI of 25-29.9, $90 \mathrm{~s}$ per frame from the skull to the mid thigh and $30 \mathrm{~s}$ per frame for the lower extremity; for a BMI of 30-35, $120 \mathrm{~s}$ per frame from the skull to the mid thigh and $30 \mathrm{~s}$ per frame for the lower extremity; for a BMI of more than 35, $180 \mathrm{~s}$ per frame from the skull to mid thigh and $60 \mathrm{~s}$ per frame for the lower extremity (Table 1).

Two protocols were used in the digital PET exams: a regularbody protocol was used for participants with a BMI of 34 or less, and a large-body protocol was used for participants with a BMI of more than 34. In the regular-body protocol, the photon counting time was $75 \mathrm{~s}$ per frame from the skull to the mid thigh (frames 110 ) and $37 \mathrm{~s}$ per frame for the lower extremity (frames 11-18). In the large-body protocol, the photon counting time was $105 \mathrm{~s}$ per frame at each bed position from the skull to the mid thigh and $45 \mathrm{~s}$ at each bed position for the lower extremity (Table 2).

\section{Data Analysis}

The comparisons were limited to participants who had identical FOVs that included either the skull to the mid thigh or the head to the toes (whole body). The mean DLP, scan time for both PET and CT exams (both FOVs, skull to mid thigh and whole body), and

TABLE 1

Variable Photon Counting Time per Frame for Analog PET Scanner for Different BMIs

\begin{tabular}{lccccc}
\hline & \multicolumn{5}{c}{ Time per frame } \\
\cline { 2 - 6 } Frames & BMI $<20$ & BMI, 20-24.9 & BMl, 25-29.9 & BMI, 30-35 & BMI > 35 \\
\hline $1-10$ & $60 \mathrm{~s}$ & $60 \mathrm{~s}$ & $90 \mathrm{~s}$ & $120 \mathrm{~s}$ & $180 \mathrm{~s}$ \\
$11-18$ & $30 \mathrm{~s}$ & $30 \mathrm{~s}$ & $30 \mathrm{~s}$ & $30 \mathrm{~s}$ & $60 \mathrm{~s}$ \\
\hline
\end{tabular}


TABLE 2

Photon Counting Time per Frame for Digital PET Scanner Based on BMls

\begin{tabular}{ccc}
\hline FOV & $\begin{array}{c}\text { Time per frame } \\
\text { in regular-body } \\
\text { protocol } \\
\text { (BMI } \leq 34)\end{array}$ & $\begin{array}{c}\text { Time per frame } \\
\text { in large-body } \\
\text { protocol (BMI > 34) }\end{array}$ \\
\hline $\begin{array}{c}\text { Skull to mid thigh } \\
\text { (frame 1-10) } \\
\text { Lower extremity } \\
(11-18)\end{array}$ & $75 \mathrm{~s}$ & $105 \mathrm{~s}$ \\
\hline
\end{tabular}

${ }^{18} \mathrm{~F}-\mathrm{FDG}$ dose were collected and compared. For statistical analyses, paired $t$ tests were applied. A $P$ value of less than 0.05 was considered significant. All analyses were performed in Microsoft Excel (version 15).

\section{RESULTS}

\section{FOV Effect on Scan Time, ${ }^{18}$ F-FDG Dose, and DLPs}

The differences between analog and digital PET/CT scanners regarding scan time, ${ }^{18} \mathrm{~F}$-FDG dose, and DLP were investigated. These data for scans that had been obtained at our institution between 2012 and 2019 were collected and compared.

The analysis revealed a shorter total scan time (from both CT and PET), a lower ${ }^{18}$ F-FDG dose, and a lower DLP for the digital scanner than for the analog scanner. In terms of skull to mid-thigh FOVs, there was a significant difference in scan time $(P<0.001 ; 37 \%$ reduction $)$ and ${ }^{18} \mathrm{~F}-\mathrm{FDG}$ dose $(P<0.001 ; 16 \%$ reduction $)$. The difference in DLPs $(P=$ $0.2 ; 8 \%$ reduction) for the Vereos when compared with the Gemini was not significant (Fig. 1; Table 3). In the head-totoe (whole-body) FOV cases, the Vereos revealed significant differences in scan time $(P<0.001 ; 33 \%$ reduction $)$, ${ }^{18} \mathrm{~F}-\mathrm{FDG}$ dose $(P<0.001 ; 13 \%$ reduction $)$, and DLP $(P<$ $0.001 ; 19 \%$ reduction) (Fig. 2 ; Table 3 ).

\section{Impact of BMI on Scan Time, ${ }^{18} \mathrm{~F}-\mathrm{FDG}$, and DLP}

The standard procedure for the administration of an ${ }^{18}$ F-FDG dose for a PET scan has been based on the weight of the participants (8). In a weight-based dosing system, body habitus is not considered and hence this system could be prone to over- or underestimation of the radiation dose that the participants may receive (9). By means of BMI, however, both weight and height are considered and the role of body habitus becomes more prominent. Therefore, BMI is thought be an effective factor in minimizing scan time, DLP, and ${ }^{18} \mathrm{~F}$ FDG dose.

To address this issue, the participant data were evaluated from 2 scanners (digital vs. analog), and a significant difference was found in total scan time between the Vereos and the Gemini $(P<0.001 ; 33 \%$ reduction; Fig. 3; Table 4). Among the BMI groups, scan time differences were the most prominent in the obese patients and the least prominent in the normal-weight patients. The lower scan times (in the digital scanner than the analog scanner) included $16 \%$ in the underweight group $(P<0.004)$, $8 \%$ in the normal-weight group ( $P=0.0014), 26 \%$ in the overweight group $(P<0.001)$, and $47 \%$ in the obese group $(P<0.001)$ (Fig. 3; Table 4)

Similarly, a significant difference was observed in the total DLP of all cases between the digital and analog systems $(P<0.001)$. However, when the cases were split into $4 \mathrm{BMI}$ groups, the decrease in DLP for the digital scanner was significant in the overweight $(P<0.001)$ and obese $(P<0.001)$ groups but not in the underweight $(P=0.8)$ group. In the normal-weight group, this trend was the opposite: the DLP was higher for the digital system than for the analog system $(P=0.08)$ (Fig. 4; Table 4). Consistently, a significantly lower value for the digital scanner (in comparison to the analog) was observed in total ${ }^{18} \mathrm{~F}-\mathrm{FDG}$ dose for all participants $(P<0.001)$, and again, significantly lower values were found for normal-weight $(P=$ $0.012)$, overweight $(P<0.001)$, and obese $(P<0.001)$ participants but not for underweight $(P=0.5)$ participants. Of note, because the ${ }^{18} \mathrm{~F}$-FDG dose had been administrated per kilogram of body weight, the major difference between the analog and the digital scans was in participants with higher BMIs (overweight and obese), most notably in participants with a BMI of more than 30. This finding suggests that these 2 groups of participants might benefit the most (lower ${ }^{18}$ F-FDG doses; Fig. 5; Table 4).

These encouraging findings merit further investigation regarding the effect of BMI on administered ${ }^{18} \mathrm{~F}-\mathrm{FDG}$ dose. Therefore, we changed the administered ${ }^{18} \mathrm{~F}$-FDG dose from weight-based to BMI-based. In this new BMI-based

TABLE 3

Mean, SD, and $P$ value for ${ }^{18}$ F-FDG Dose, Scan Time, and DLP for Gemini Vs. Vereos PET/CT Scanner Regarding Different FOVs

\begin{tabular}{|c|c|c|c|c|c|c|c|c|c|}
\hline \multirow[b]{2}{*}{ FOV } & \multicolumn{3}{|c|}{${ }^{18} \mathrm{~F}-\mathrm{FDG}$ dose (MBq) } & \multicolumn{3}{|c|}{ Scan time (s) } & \multicolumn{3}{|c|}{ DLP (mGy/cm) } \\
\hline & Gemini & Vereos & $P$ & Gemini & Vereos & $P$ & Gemini & Vereos & $P$ \\
\hline $\begin{array}{l}\text { Skull to } \\
\text { mid thigh }\end{array}$ & 443.26 (91.95) & $370.00(46.46)$ & $<0.001$ & $1,342.24(510)$ & 836.40 (139.2) & $<0.001$ & 463.33 (162.14) & 424.38 (115) & NS \\
\hline $\begin{array}{l}\text { Whole } \\
\text { body }\end{array}$ & 411.99 (95.30) & $355.94(62.24)$ & $<0.001$ & 1,619.95 (595.8) & 1,083.24 (180.6) & $<0.001$ & 702.22 (261.36) & $567.46(144.28)$ & $<0.001$ \\
\hline
\end{tabular}

NS = not significant.

Data are mean followed by SD in parentheses. $P$ values were determined by $t$ test. 


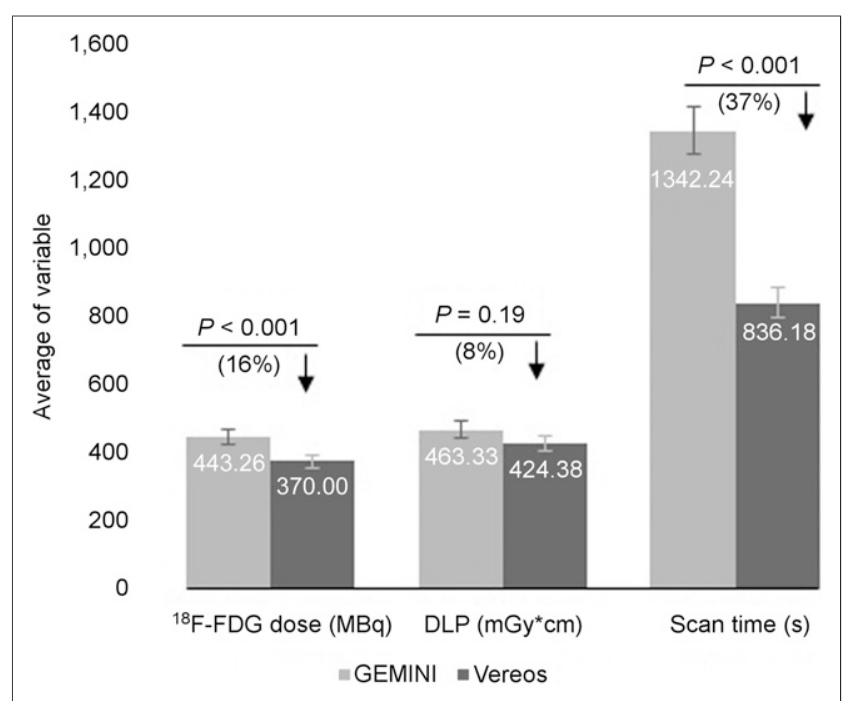

FIGURE 1. Skull-to-mid-thigh FOV indicates significant difference in scan time and ${ }^{18} \mathrm{~F}-\mathrm{FDG}$ dose between Vereos and Gemini. No significant difference in DLP was observed. $P$ values, percentages of change (differences), and decreases $(\downarrow)$ in corresponding values are indicated. Error bars are indicated as $5 \%$.

system (20 participants), the administered ${ }^{18} \mathrm{~F}$-FDG dose was $222 \mathrm{MBq}(6 \mathrm{mCi})$ for participants with a BMI of less than $25,296 \mathrm{MBq}(8 \mathrm{mCi})$ for participants with a BMI of 26-34, and $370 \mathrm{MBq}(10 \mathrm{mCi})$ for participants with a BMI of more than 35 in the digital system. The data on the administered ${ }^{18} \mathrm{~F}-\mathrm{FDG}$ dose from the Gemini, the Vereos weight-based dose, and the Veroes BMI-based dose were collected and compared with one another. The results show

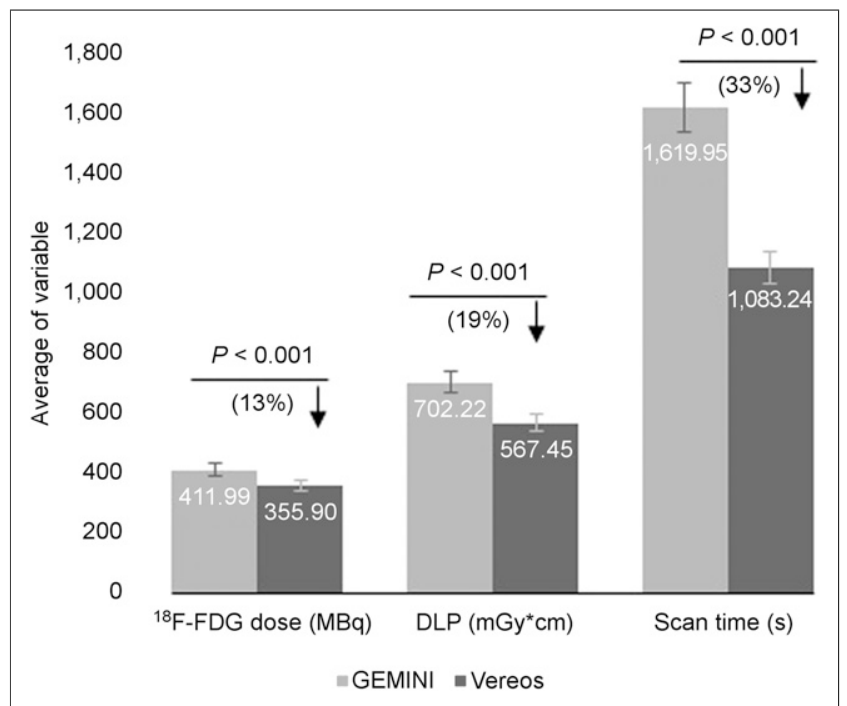

FIGURE 2. Head-to-toe (whole body) FOV for Vereos indicates significant difference in scan time compared with Gemini. $P$ values, percentages of changes (differences), and decreases $(\downarrow)$ in corresponding values are indicated. Error bars are indicated as $5 \%$.

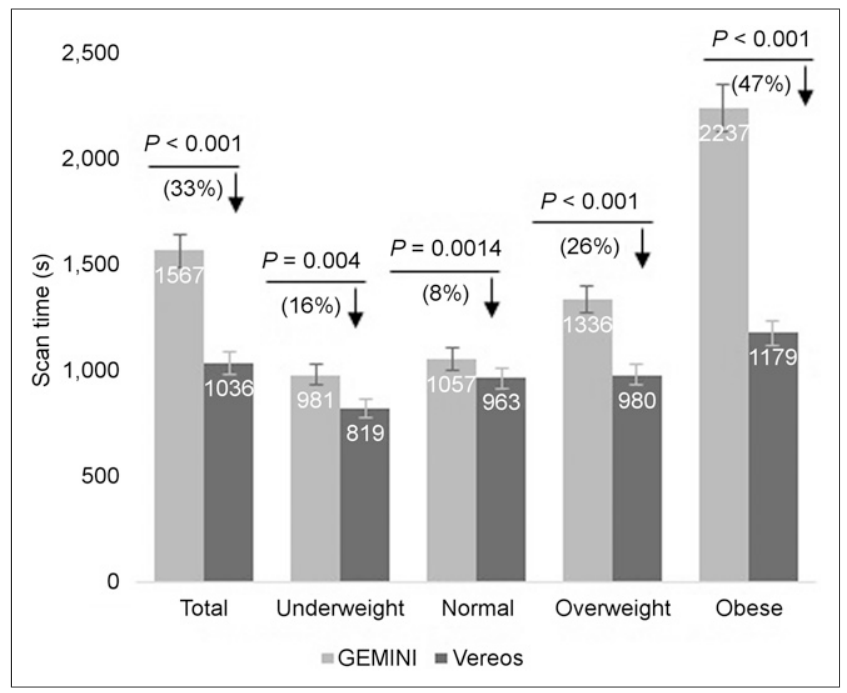

FIGURE 3. Scan time for digital and analog PET/CT systems according to BMI group. Total scan time shows significant difference between the 2 scanners $(P<0.001 ; 33 \%$ reduction). Largest difference was among obese patients, and least difference was among normal-weight patients. $P$ values, percentages of changes (differences), and decreases $(\downarrow)$ in corresponding values are indicated. Error bars are indicated as $5 \%$.

a significant difference between the Gemini weight-based dose and the Vereos weight-based dose $(P<0.001 ; 34 \%$ reduction) and between the Vereos weight-based dose and the Vereos BMI-based dose $(P<0.001 ; 22 \%$ reduction $)$ (Fig. 6; Table 5). However, the new dosing system is an ongoing research project designed to reduce the radiation dose to participants.

\section{Image Quality}

Review of the image quality demonstrated a mean score of 4.0 for the analog scanner and 4.5 for the digital scanner. Overall, the images appeared less noisy on the digital scanner than on the analog scanner.

\section{DISCUSSION}

Historically, the early idea of PET was developed in 1950 and the first scanner was innovated in 1970 at Washington University in Saint Louis, MO (3). From the early singleslice detector design to the commercial scanners of today, the benefits of PET/CT in oncologic studies cannot be denied, but there have always been aspirations of reducing the ${ }^{18} \mathrm{~F}-\mathrm{FDG}$ dose and scan time. The single pair of detectors in planar imaging has evolved into the current one-to-one solid detectors in PET, which has led to advanced acquisition electronics, data processing, and image analysis over the past 50 years (3). The latest improvement in PET/CT scanners has been the digital photon-counting technique, which promises a shorter scan time and a lower radiation dose while improving image quality and small-lesion detectability. 


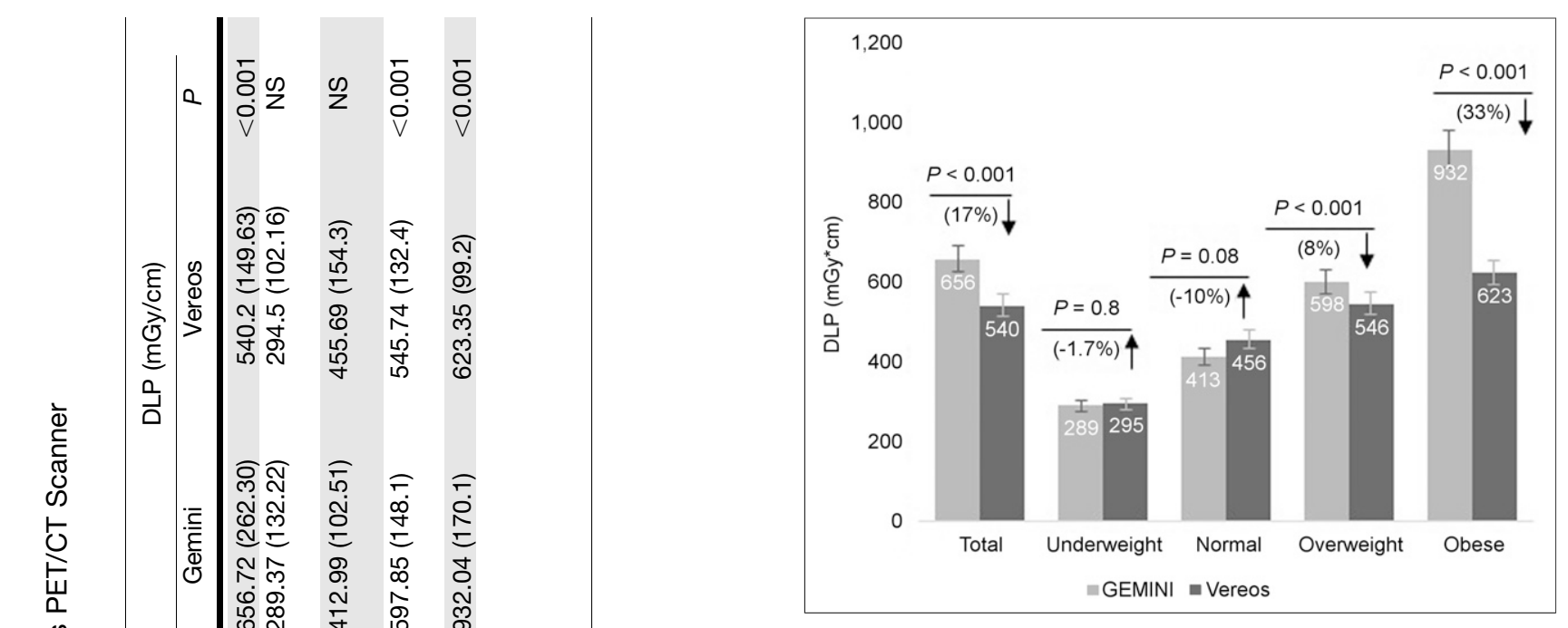

FIGURE 4. DLP for digital and analog PET/CT systems according to BMI group. Significant difference was observed in overweight and obese groups but not in underweight or normalweight groups. $P$ values, percentages of changes (differences), and increases $(\uparrow)$ or decreases $(\downarrow)$ in corresponding values are indicated. Error bars are indicated as $5 \%$.

Multiple studies $(10,11)$ have reported the superiority of digital PET/CT systems over analog scanners respecting improved small-lesion detectability and image quality $(10,11)$. However, to our knowledge, no study has addressed the differences between digital and analog PET/CT scanners with respect to scan time, radiation dose from administered dose, and radiation dose from the CT portion of the scan. In this study, scan time, ${ }^{18}$ F-FDG dose, and DLP were evaluated in digital and analog PET/CT scanners, and significant differ-

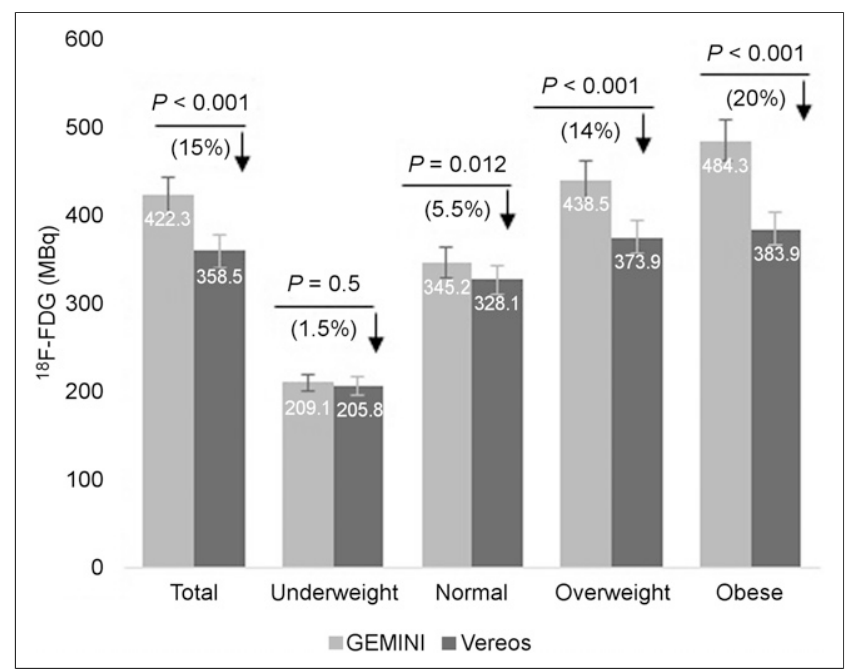

FIGURE 5. ${ }^{18} \mathrm{~F}-\mathrm{FDG}$ dose for digital and analog PET/CT systems according to BMI group. Significantly lower doses were observed in normal-weight, overweight, and obese groups but not in underweight group. $P$ values, percentages of changes (differences), and decreases $(\downarrow)$ in corresponding values are indicated. Error bars are indicated as $5 \%$. 


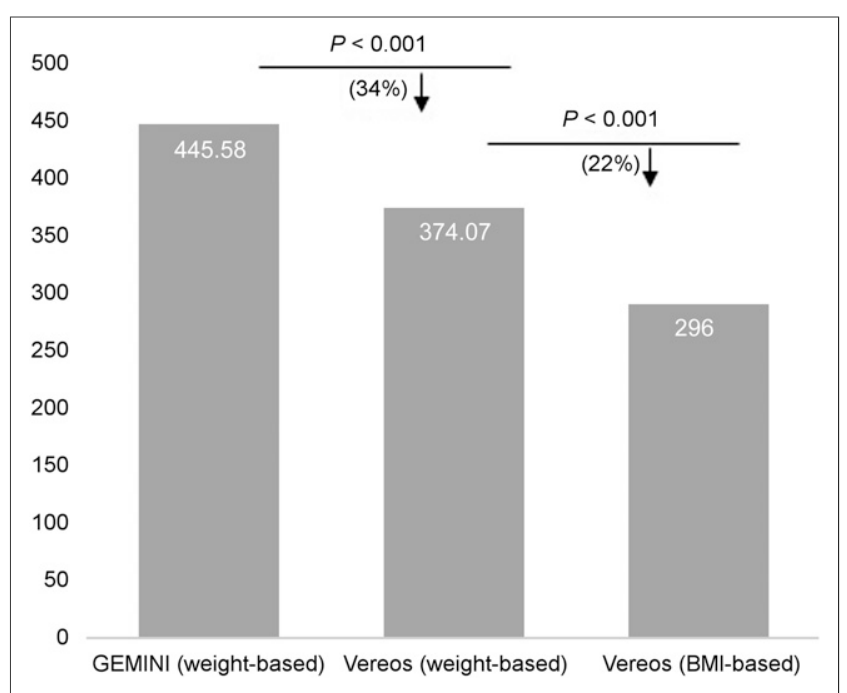

FIGURE 6. Comparisons between Gemini weight-based dosing, Vereos weight-based dosing, and Vereos BMI-based dosing. Significant difference is seen between analog and digital weight-based dosing systems and between digital weightbased and digital BMI-based dosing systems. $P$ values, percentages of changes (differences), and decreases $(\downarrow)$ in corresponding values are indicated. Error bars are indicated as $5 \%$.

ences in these factors were observed between the digital and analog scanners. In line with expectations, scan time and radiation dose were lower in digital than in analog PET/ CT scanners (12). With a few exceptions (underweight patients), results from all patients indicated a significantly shorter scan time and reduced radiation dose in the digital scanner than in the analog scanner (Fig. 3; Table 4).

The DLP in the skull-to-mid-thigh FOV showed no significant difference that could be raised from the differences in calculation methods between digital and analog scanners. The digital DLP is calculated using the sum of the radiation dose from both the scout and the slices. However, for the analog scanner, the DLP could use the sum of the radiation from only the slices since the scout was not available. This in theory should give the digital scanner a higher DLP; however,

TABLE 5

Differences Between Gemini Weight-Based and Vereos Weight-Based Dose System $(P<0.001)$ and Between Vereos Weight-Based Dose System and Vereos BMIBased Dose System $(P<0.001)$

\begin{tabular}{|c|c|c|c|c|c|}
\hline $\begin{array}{c}\text { Gemini } \\
\text { weight- } \\
\text { based }\end{array}$ & $P$ & $\begin{array}{c}\text { Vereos } \\
\text { weight-based }\end{array}$ & $P$ & $\begin{array}{c}\text { Vereos } \\
\text { BMI- } \\
\text { based }\end{array}$ & $P$ \\
\hline $\begin{array}{l}445.58 \\
(72.57)\end{array}$ & $<0.001$ & $\begin{array}{l}374.07 \\
(27.43)\end{array}$ & $<0.001$ & $\begin{array}{c}296 \\
(56.75)\end{array}$ & $<0.001$ \\
\hline
\end{tabular}

Data are mean ${ }^{18} \mathrm{~F}-\mathrm{FDG}$ dose in megabecquerels, followed by SD in parentheses. the new iDose iterative reconstruction technique is able to maintain image quality with a lower radiation dose. Our evaluation likely underestimates the radiation savings since the scout is not included in the analog DLP calculation. In terms of different BMI groups, the DLP and ${ }^{18}$ F-FDG dose values were not beneficial for underweight participants, for 2 potential reasons. The first is the mentioned reason for variations in DLP calculations between digital and analog scanners. The second comes from undersampling in underweight groups due to exclusion criteria (most of the participants in these groups were excluded from further analyses because there was more than a $10 \%$ variation in their BMIs).

Since the administered ${ }^{18} \mathrm{~F}$-FDG dose was weight-based, the results illustrate that reducing the ${ }^{18} \mathrm{~F}-\mathrm{FDG}$ dose would not be beneficial to underweight participants but would be beneficial to normal-weight, overweight, and obese participants. Per our findings, the dosing system has been changed to a BMI-based system instead of weight-based at our center. In this new BMI-based system (20 participants), the administered ${ }^{18} \mathrm{~F}-\mathrm{FDG}$ dose is $222 \mathrm{MBq}(6 \mathrm{mCi})$ for participants with a BMI of less than $25,296 \mathrm{MBq}$ (8 $\mathrm{mCi}$ ) for participants with a BMI of $26-34$, and $370 \mathrm{MBq}$ (10 $\mathrm{mCi})$ for participants with a BMI of more than 35 for the Vereos PET/CT system (Fig. 6; Table 5).

Some limitations should be considered regarding the interpretation and generalization of our findings. First, this was a single-institution study. Second, it is important to remember that in a digital scanner, DLP is not calculated the same way as in an analog scanner but that DLP has been used for evaluation of radiation in CT, and as this study was retrospective, this parameter could not be edited. Third, since this was a retrospective study, our findings need to be reevaluated prospectively. In addition, lesion detectability could not be assessed because of the different time points in the scans of each patient. However, this has been assessed in a recent study in which patients had both scans after a single ${ }^{18}$ F-FDG injection and confirmed that the digital scanner demonstrated superior small-lesion detection (13). Fourth, we compared digital and analog PET/CT scanners from the same manufacturer. Future research could compare data acquired from a digital PET/CT scanner from multiple manufacturers, again comparing the scan time, ${ }^{18}$ F-FDG dose, and DLP. Finally, we did not find beneficial effects for underweight participants, and it is worth noting that 2 potential intrinsic factors could be involved here: the first is that in this short period between the first and followup scans for each patient, a big shift in the weight of each participant would be unlikely; the second is that, yet, these small changes would significantly affect the "percentage of weight change" in the underweight patients but not in the overweight or obese patients. Therefore, undersampling for the underweight patients might be a caveat of our study. Expanding this analysis to a larger population of underweight patients might help clarify this conundrum. 


\section{CONCLUSION}

Compared with the Gemini analog PET/CT system, the Vereos digital PET/CT scanner provides improved image quality with the benefits of shorter scan time, lower radiation exposure dose, and lower administered ${ }^{18} \mathrm{~F}-\mathrm{FDG}$ dose, which lead to a lower radiation dose to the technologist and the public. On the basis of this study, we conclude that the digital $\mathrm{PET} / \mathrm{CT}$ scanner is a beneficial molecular imaging modality in view of its lower radiation dose and shorter scan time.

\section{DISCLOSURE}

No potential conflict of interest relevant to this article was reported.

\section{ACKNOWLEDGMENTS}

We gratefully acknowledge the support of Drs. Reza Ghasemi and Austin Turner in manuscript writing and editing.

\section{REFERENCES}

1. Weber WA, Grosu AL, Czernin J. Technology Insight: advances in molecular imaging and an appraisal of PET/CT scanning. Nat Clin Pract Oncol. 2008;5:160-170.

2. Truly digital PET imaging: Philips proprietary Digital Photon Counting technology. UserManual.wiki website. https://usermanual.wiki/Philips/882446.1363632199/view. Published 2016. Accessed October 13, 2020.

3. Jones T, Townsend D. History and future technical innovation in positron emission tomography. J Med Imaging (Bellingham). 2017;4:011013.
4. Jaime M, Botkin C, Frye S, et al. Evaluation of digital PET imaging Vereos PET/ CT [abstract]. J Nucl Med. 2019;60(suppl 1):2059.

5. Why - and how-digital PET is better than analog. Imaging Technology News website. https://www.itnonline.com/content/blogs/greg-freiherr-industry-consultant/whyand-how-digital-pet-better-analog. Published October 17, 2020. Accessed October 13, 2020.

6. Truly digital PET imaging: Philips proprietary Digital Photon Counting technology. Philips InCenter website. http://incenter.medical.philips.com/doclib/enc/ 11024203/452299106341_Digital_PET_Technical_Overview_HR.pdf\%3ffunc\% 3ddoc.Fetch\%26nodeid\%3d11024203. Published 2014. Accessed October 13, 2020.

7. iDose $^{4}$ iterative reconstruction technique. Philips InCenter website. http://incenter. medical.philips.com/doclib/enc/fetch/2000/4504/577242/577249/586938/587315/ iDose4_-_Whitepaper_-_Technical_-Low_Res.pdf\%3fnodeid\%3d8432599\%26vernum \%3d-2. Published 2011. Accessed October 13, 2020.

8. Karakatsanis NA, Fokou E, Tsoumpas C. Dosage optimization in positron emission tomography: state-of-the-art methods and future prospects. Am J Nucl Med Mol Imaging. 2015;5:527-547.

9. Nagaki A, Onoguchi M, Matsutomo N. Patient weight-based acquisition protocols to optimize ${ }^{18}$ F-FDG PET/CT image quality. J Nucl Med Technol. 2011;39:72-76.

10. Nguyen NC, Vercher-Conejero JL, Sattar A, et al. Image quality and diagnostic performance of a digital PET prototype in patients with oncologic diseases: initial experience and comparison with analog PET. J Nucl Med. 2015;56:1378-1385.

11. López-Mora DA, Flotats A, Fuentes-Ocampo F, et al. Comparison of image quality and lesion detection between digital and analog PET/CT. Eur J Nucl Med Mol Imaging. 2019;46:1383-1390.

12. SNMMI position statement on dose optimization for nuclear medicine and molecular imaging procedure. SNMMI website. http://s3.amazonaws.com/ rdcms-snmmi/files/production/public/docs/SNM_Position_Statement_on_Dose_ Optimization_FINAL_June_2012.pdf. Published June 2012. Accessed October $13,2020$.

13. Koopman D, van Dalen JA, Stevens H, Slump CH, Knollema S, Jager PL. Performance of digital PET compared with high-resolution conventional PET in patients with cancer. J Nucl Med. 2020;61:1448-1454. . 